\title{
Ultrasonido transvaginal para la detección preoperatoria de endometriosis profunda en pacientes con dolor pélvico crónico
}

\author{
Aníbal Scarella C. ${ }^{1,2}$, Luigi Devoto C. ${ }_{2}^{2}$,Claudio Villarroel Q. ${ }^{2}$, Nicolás Inzunza P. a, \\ Felipe Quilodrán R. ${ }^{\text {, Hugo Sovino S. }}{ }^{2}$ \\ 1 Departamento Ginecología y Obstetricia, Facultad de Medicina, Universidad de Valparaíso. ${ }^{2}$ Instituto de Investigacio- \\ nes Materno Infantil, Facultad de Medicina, Universidad de Chile.
}

a Alumnos, Escuela de Medicina, Universidad de Valparaíso.

\section{RESUMEN}

Antecedentes: La endometriosis profunda (EP) es una enfermedad caracterizada por lesiones que penetran $>5 \mathrm{~mm}$ la superficie del peritoneo pélvico. Representa la forma más sintomática de la enfermedad y un importante desafío quirúrgico. La resonancia magnética, la ultrasonografía transrectal y la ultrasonografía transvaginal (USTV), se consideran métodos de diagnóstico adecuados, no obstante, este último ofrece ventajas de accesibilidad, costo-efectividad y tolerancia. Objetivo: Implementar y determinar la capacidad de la USTV para detectar EP comparando los hallazgos con la laparoscopía. Métodos: Estudio transversal de prueba diagnóstica que incluye 57 pacientes con sospecha de endometriosis e indicación quirúrgica. Se realizó la USTV, evaluando la presencia, localización, tamaño y grado de infiltración de la EP. Los resultados ecográficos se compararon con los hallazgos quirúrgicos e histológicos. Resultados: La EP se confirmó quirúrgica e histológicamente en 35/57 pacientes. Se identificó endometriosis ovárica (EO) y EP en la laparoscopía en 35 y 31 de las mujeres, respectivamente. Para el diagnóstico de la EP, la USTV tuvo una sensibilidad (S) de $94,3 \%$, especificidad (E) 100\%, valor predictivo positivo (VPP) de 100\%, valor predictivo negativo (VPN) del 91,7\% y un exactitud (Ex) de 96,5\%. Para el diagnóstico EP sobre los ligamentos uterosacros, la S, E, VPP y VPN fue: $85,7 \%, 100 \%, 100 \%$ y $98 \%$ respectivamente. Para el diagnóstico de EP con compromiso intestinal, la S, E, VPP y VPN fue 100\%. Conclusión: Estos hallazgos muestran que USTV es un examen adecuado para la evaluación de la EP y confirma su importancia para definir una estrategia quirúrgica y consejería preoperatoria.

PALABRAS CLAVE: Endometriosis profunda, ultrasonografía transvaginal, detección preoperatoria

\section{SUMMARY}

Background: Deep endometriosis (DE) is defined arbitrarily as endometriosis infiltrating the peritoneum by $>5 \mathrm{~mm}$. Represents the most symptomatic disease and a major surgical challenge. Currently, MRI, transrectal ultrasonography and transvaginal ultrasonography (USTV), are considered appropriate diagnostic methods, however, the latter offers advantages in terms of accessibility, cost-effectiveness and tolerance. Objective: To implement and assess the ability of the USTV for detect DE having laparoscopy as the gold standard. Methods: Cross-sectional diagnostic test study that included 57 patients with suspected endometriosis and surgical indication. USTV was performed by a single operator assessing the presence, location, size and degree of infiltration of the DE. The sonographic findings were compared with surgical and histological findings. Results: The DE was confirmed surgically and histologically in 35/57 patients. Ovarian endometriosis (OE) and DE were identified and at laparoscopy in 35 and 31 women, respectively. 
For the diagnosis of DE, the USTV had a sensitivity (S) of $94.3 \%$, specificity (E) $100 \%$, positive predictive value (PPV) of $100 \%$, negative predictive value (NPV) of $91.7 \%$ and accuracy (A) of $96.5 \%$. For diagnostic DE of the uterosacral ligaments, S, E, PPV and NPV were: $85.7 \%, 100 \%, 100 \%$ and $98 \%$, respectively. For the diagnosis of DE with intestinal involvement, S, E, PPV and NPV was 100\%. Conclusion: These findings show that USTV is adequate technique for the evaluation of the EP and confirms the importance to define a surgical strategy and preoperative counseling.

\section{KEY WORDS: Deep infiltrating endometriosis, transvaginal ultrasound, preoperative diagnosis}

\section{INTRODUCCIÓN}

La endometriosis, es una enfermedad frecuente, que afecta al $6-10 \%$ de las mujeres en edad reproductiva y entre un $50-60 \%$ de las mujeres con dolor pélvico crónico o con infertilidad $(1,2)$. La endometriosis se puede clasificar en endometriosis superficial, profunda $(E P)$ y ovárica $(E O)(3,4)$. La EP es una entidad histológicamente específica, definida como lesiones endometriósicas que se extienden más de $5 \mathrm{~mm}$ bajo el peritoneo (3). Habitualmente, la EP representa la forma más sintomática de la enfermedad, cuyo manejo representa un desafío quirúrgico, especialmente cuando hay afección intestinal $(5,6)$.

La EP es difícil de evaluar mediante el examen físico, identificándose el compromiso intestinal sólo en un $40-68 \%$ de los casos (7). En los últimos años, se han desarrollado distintas técnicas de imagen que permiten una adecuada planificación y consejería preoperatoria de la paciente. Dentro de ellas destacan la ultrasonografía transvaginal (USTV) y transrectal (USTR) y la resonancia magnética (RM). Éstas exhiben tasas de sensibilidad y especificidad adecuadas (8-11), siendo la RM la técnica clásica de elección para el diagnóstico de la EP (12).

Recientemente, publicaciones han demostrado que la ultrasonografía transvaginal con preparación intestinal (USTV-PI), alcanzan niveles predictivos para endometriosis superiores a la RM (13). En vista de estos resultados y de los menores costos asociados, diversos autores consideran USTV como de primera línea en el diagnóstico de la EP $(6,13,14)$. A pesar de lo anterior, dicha técnica hoy se ejecuta únicamente en un número limitado de centros médicos alrededor del mundo, y en Chile aún no hay experiencias publicadas.

El objetivo de este estudio es implementar y evaluar la capacidad diagnóstica de la USTV-PI para la detección de EP en pacientes con sospecha clínica de endometriosis.

\section{PACIENTES Y MÉTODOS}

Estudio multicéntrico transversal de pruebas diagnósticas realizado entre septiembre de 2011 y septiembre de 2012 en el Instituto de Investigación
Materno Infantil (IDIMI) de la Facultad de Medicina de la Universidad de Chile, y en el Centro de Reproducción Humana de la Facultad de Medicina de la Universidad de Valparaíso. El estudio cuenta con la aprobación del Comité de Ética del Servicio de Salud Metropolitano Central.

Se incluyó a todas las pacientes con dolor pélvico crónico (mayor o igual a 6 meses de duración) y/o con sospecha de endometriosis, con indicación de cirugía. Se excluyen las pacientes posmenopáusicas, pacientes con cirugías previas de colon y/o sigmoides y pacientes con otra causa conocida de dolor pélvico.

Previo al examen, todas las pacientes se sometieron a una preparación intestinal a fin eliminar los residuos fecales y gases presentes en el rectosigmoide, mejorando la capacidad de visualización de lesiones endometriósicas pélvicas. Según el protocolo de Goncalves y cols (6), se indicó: (a) laxante suave oral ( 25 gotas de Guttalax $\AA$ ) entre las 8:00 am y las 2:00 pm el día antes del examen (b) dieta baja en residuos en la víspera y el día del examen y (c) enema rectal (Fleet Enema ${ }^{\circledR}$ ) aproximadamente 1-2 horas antes del examen. Durante la exploración, se aplicó 20-40 cc de gel de ultrasonido en el fórnix vaginal.

Cada examen se interpreta en tiempo real y es documentado en fotografías. El examen consta de una evaluación transvaginal realizada por un único evaluador (AS), realizada con un ecógrafo Medison, SonoaceX6 con un transductor de 5 a 9-MHz. Se evaluaron en forma sistemática los siguientes órganos o estructuras que se ordenan de ventral a dorsal: vejiga, repliegue vesicouterino, meatos ureterales, útero, ovarios, fondo de saco de Douglas, espacio retrocervical, ligamentos uterosacros (LUS), tabique rectovaginal y fórnix vaginal posterior. También se evaluó el rectosigmoide desde el borde anal hasta 20-25 cm sobre el anterior, examinando en detalle las distintas capas de la pared según protocolo de Goncalves y cols (6).

En resumen, se evalúa en forma sistemática, desde el exterior hacia el lumen interno pasando por las siguientes capas (a) serosa (línea delgada hiperecoica); (b) muscularis propria (dos líneas hipoecoicas separadas por una fina línea hiperecoica); (c) submucosa (capa hiperecoica); (d) mucosa 
(hiperecoica) (Figura 1). El hallazgo de EP se estableció ante la visualización de una o más lesiones gruesas, nodulares, hipoecoicas ubicadas en las áreas descritas.

Identificada una lesión, la descripción incluyó: A. Tamaño, consignando los 3 ejes en su punto mayor. B. Signos de adherencias e infiltración a los sitios mencionados (movilizando el transductor se evaluó como se deslizan las estructuras). C. En el caso de las lesiones intestinales se determinó las capas intestinales comprometidas, el porcentaje de la circunferencia afectada y la distancia desde el borde anal hasta la lesión, midiendo la distancia entre la lesión y la reflexión peritoneal estimada a 8 $\mathrm{cm}$ desde el borde anal.

Una vez realizado el examen, las pacientes se sometieron a cirugía según la indicación del tratante, independiente de los hallazgos de la USTV. Los hallazgos de la laparoscopia o cirugía abierta se registraron en forma protocolizada. Se solicitó consignar en forma ciega para el ecografista el número, tamaño, grado de adherencias e infiltración a órganos pélvicos.

Para el análisis estadístico se compararon los hallazgos ecográficos con los operatorios. Se consideró como estándar dorado o patrón de referencia para endometriosis el informe de la biopsia y/o la visualización directa por el cirujano (16). Para el análisis de los resultados se utilizó el software SPSS v16.0. Se calcularon pruebas de sensibilidad, especificidad, valor predictivo positivo y negativo.

\section{RESULTADOS}

Durante el periodo estudiado, se evaluaron en forma consecutiva 100 pacientes con indicación quirúrgica por dolor pélvico y/o sospecha de endometriosis. De ellas, 22 tienen pendiente la cirugía, 21 pospusieron la cirugía para someterse a una técnica de reproducción asistida. Del total, finalmente se incluyeron 57 pacientes sometidas a cirugía.

De las incluidas se identificaron 31 pacientes con endometriosis ovárica y 35 con EP. En estas últimas, se distribuyeron de la siguiente forma: 28 pacientes con EP retrocervical c/s compromiso intestinal y 7 con EP de los LUS sin compromiso retrocervical. Además, hubo 3 pacientes con EP prevesical, todas presentando EP en otros sitios de la pelvis. Las características epidemiológicas de las pacientes se describen en la Tabla I.

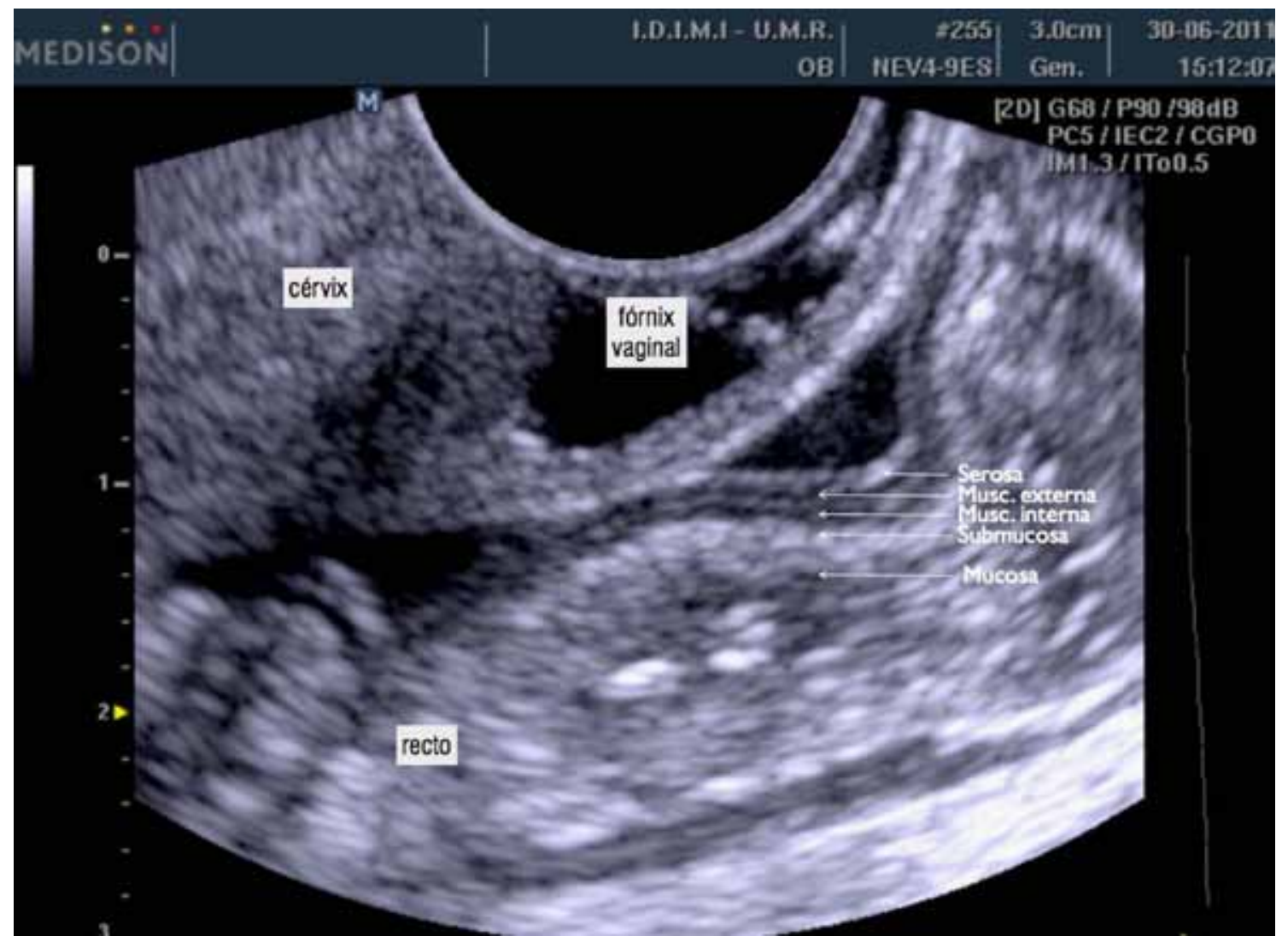

Figura 1. Evaluación ultrasonográfica del tabique recto-vaginal. 
Tabla I

\section{CARACTERÍSTICA DE LAS PACIENTES CON DOLOR PÉLVICO CRÓNICO SOMETIDAS A CIRUGÍA}

\begin{tabular}{lc}
\hline Característica & $\begin{array}{c}\text { Número de } \\
\text { pacientes }(\%)\end{array}$ \\
\hline Infertilidad & $29 / 57(50,8)$ \\
Nuliparidad & $30 / 57(52,6)$ \\
Dolor cíclico moderado-severo & $50 / 57(87,8)$ \\
Dispareunia & $30 / 57(52,6)$ \\
\hline
\end{tabular}

La capacidad diagnóstica según la ubicación anatómica se resume en la Tabla II. La USTV-PI identificó a $33(94,3 \%)$ de las 35 pacientes con EP. Del total de pacientes con EP, encontramos falsos negativos en pacientes con EO (1 caso), EP retrocervical (1 caso), EP de LUS aislada (1 casos) y EP prevesical (1 caso).

A pesar de lo anterior, se obtuvo una exactitud $>$ $90 \%$ y una especificidad del $100 \%$ en cada una de las regiones evaluadas. Además, la técnica ecográfica detectó todas las pacientes con endometriosis retrocervical con compromiso intestinal (24 de 32 pacientes con EP) logrando una exactitud del $100 \%$ en este parámetro.

\section{DISCUSIÓN}

Nuestro estudio muestra la exactitud de la USTV-PI para la detección de EP comparada con la laparoscopia. Corresponde a la primera casuística nacional publicada dando cuenta de la necesidad de detección preoperatoria de la endometriosis.

Una adecuada prueba diagnóstica permite orientar a los especialistas sobre la necesidad de realizar la cirugía, planificar el momento oportuno, el abordaje óptimo, así como informar a las pacientes sobre el resultado probable de la cirugía $(6,7,13)$. Lo anterior, cobra particular importancia en casos de EP, al representar la forma más sintomática y desafiante desde el punto de vista quirúrgico $(3,17,18)$.

La clínica y el examen físico constituyen la primera aproximación en la paciente con sospecha de EP $(13,14,19)$, sin embargo, a pesar de ser parte integral de la evaluación de las pacientes con dismenorrea o dolor pélvico, no siempre tienen una adecuada correlación entre los hallazgos y el avance de la enfermedad $(7,13)$. Así, el uso de técnicas complementarias como la RM y la USTR y la USTV han surgido como alternativas para la aproximación diagnóstica de la paciente con sospecha de EP. En estudios recientes, la USTV ha demostrado ser equivalente (8), o incluso superior que la RM para la detección de esta patología (13).

Al igual que otros autores, demostramos la alta capacidad diagnóstica del USTV en estudio de la paciente con EP $(6,13,14)$. Nuestros resultados son comparables a una revisión sistemática y meta-análisis que evaluó la precisión de la USTV para la detección de EP intestinal estimando la sensibilidad y especificidad del $91 \%$ y $98 \%$, respectivamente (20).

Estos resultados destacan el valor de la USTV en el diagnóstico preoperatorio de la EP al permitir una adecuada detección y ubicación topográfica de la enfermedad. La identificación del compromiso de la pared intestinal cobra mayor relevancia en cuanto permite la conformación de equipos multidisciplinarios y la oportuna derivación de la paciente a centros especializados en el manejo de esta patología, junto con la adecuada consejería preoperatoria.

La implementación de la USTV en los centros de atención ginecológica y su incorporación en los programas de formación de los futuros ginecólogos, aumentará la capacidad de reproducir estos resultados y la calidad de la evaluación primaria de las pacientes con dolor pélvico.

Tabla II

\section{LOCALIZACIÓN DE LA ENDOMETRIOSIS SEGÚN LA USTV-PI Y SU CORRELACIÓN CON LOS HALLAZGOS LAPAROSCÓPICOS}

\begin{tabular}{lcrrr}
\hline & & Ubicación & \\
& EP & EO & EP-RC & EP-LUS \\
\hline S (\%) & $33 / 35(94,3)$ & $30 / 31(96,8)$ & $27 / 28(96,4)$ & $6 / 7(85,7)$ \\
E (\%) & $22 / 22(100)$ & $26 / 26(100)$ & $29 / 29(100)$ & $50 / 50(100)$ \\
VPP (\%) & $33 / 33(100)$ & $30 / 30(100)$ & $27 / 27(100)$ & $6 / 6(100)$ \\
VPN (\%) & $22 / 24(91,7)$ & $26 / 27(96,6)$ & $29 / 30(96,7)$ & $50 / 51(98,0)$ \\
\hline
\end{tabular}

$\mathrm{EP}=$ endometriosis profunda; $\mathrm{EO}=$ endometriosis ovárica; $\mathrm{RC}=$ retrocervical; $\mathrm{LUS}=$ ligamento útero-sacro. 


\section{CONCLUSIÓN}

Los hallazgos de nuestra investigación muestran que USTV es un examen adecuado para la evaluación de la EP y confirma la importancia de esta técnica para definir una estrategia quirúrgica y consejería preoperatoria.

\section{REFERENCIAS}

1. Eskenazi B, Warner ML. Epidemiology of endometriosis. Obstet Gynecol Clin North Am 1997;24:235-58.

2. McLeod BS, Retzloff MG. Epidemiology of endometriosis: an assessment of risk factors. Clin Obstet Gynecol 2010;53:389-96.

3. Koninckx PR, Meuleman C, Demeyere S, Lesaffre E, Cornillie FJ. Suggestive evidence that pelvic endometriosis is a progressive disease, whereas deeply infiltrating endometriosis is associated with pelvic pain. Fertil Steril 1991;55:759-65.

4. Donnez J, Nisolle M, Smoes P, Gillet N, Beguin S, Casanas-Roux F. Peritoneal endometriosis and "endometriotic" nodules of the rectovaginal septum are two different entities. Fertil Steril 1996;66:362-8.

5. Chapron C, Chopin N, Borghese B, Malartic C, Decuypere $\mathrm{F}$, Foulot $\mathrm{H}$. Surgical management of deeply infiltrating endometriosis: an update. Ann N Y Acad Sci 2004;1034:326-37.

6. Goncalves MO, Dias JA, Jr., Podgaec S, Averbach M, Abrao MS. Transvaginal ultrasound for diagnosis of deeply infiltrating endometriosis. Int J Gynaecol Obstet 2009;104:156-60.

7. Chapron C, Dubuisson JB, Pansini V, Vieira M, Fauconnier A, Barakat $\mathrm{H}$, et al. Routine clinical examination is not sufficient for diagnosing and locating deeply infiltrating endometriosis. J Am Assoc Gynecol Laparosc 2002;9:115-9.

8. Bazot M, Lafont C, Rouzier R, Roseau G, ThomassinNaggara I, Darai E. Diagnostic accuracy of physical examination, transvaginal sonography, rectal endoscopic sonography, and magnetic resonance imaging to diagnose deep infiltrating endometriosis. Fertil Steril 2009;92:1825-33.

9. Chapron C, Dumontier I, Dousset B, Fritel X, Tardif D, Roseau G, et al. Results and role of rectal endoscopic ultrasonography for patients with deep pelvic endometriosis. Hum Reprod 1998;13:2266-70.
10. Kinkel K, Chapron C, Balleyguier C, Fritel X, Dubuisson JB, Moreau JF. Magnetic resonance imaging characteristics of deep endometriosis. Hum Reprod 1999;14:1080-6.

11. Bazot M, Detchev R, Cortez A, Amouyal P, Uzan S, Darai $\mathrm{E}$. Transvaginal sonography and rectal endoscopic sonography for the assessment of pelvic endometriosis: a preliminary comparison. Hum Reprod 2003;18:1686-92.

12. Chamie LP, Pereira RM, Zanatta A, Serafini PC. Transvaginal US after bowel preparation for deeply infiltrating endometriosis: protocol, imaging appearances, and laparoscopic correlation. Radiographics 2010;30:1235-49.

13. Abrao MS, Goncalves MO, Dias JA, Jr., Podgaec S, Chamie LP, Blasbalg R. Comparison between clinical examination, transvaginal sonography and magnetic resonance imaging for the diagnosis of deep endometriosis. Hum Reprod 2007;22:3092-7.

14. Piketty M, Chopin N, Dousset B, Millischer-Bellaische $A E$, Roseau G, Leconte $M$, et al. Preoperative work-up for patients with deeply infiltrating endometriosis: transvaginal ultrasonography must definitely be the first-line imaging examination. Hum Reprod 2009;24:602-7.

15. Kennedy S, Bergqvist A, Chapron C, D'Hooghe T, Dunselman G, Greb R, et al. ESHRE guideline for the diagnosis and treatment of endometriosis. Hum Reprod 2005;20:2698-704.

16. The investigation and management of endometriosis. Green Top Guideline; The Royal College of Obstetricians and Gynaecologists; 2006.

17. Cornillie FJ, Oosterlynck D, Lauweryns JM, Koninckx PR. Deeply infiltrating pelvic endometriosis: histology and clinical significance. Fertil Steril 1990;53:978-83.

18. Abrao MS, Podgaec S, Dias JA, Jr., Goncalves MO. Diagnosis of rectovaginal endometriosis. Hum Reprod 2008;23:2386. Author reply 2386-7.

19. Falcone T, Lebovic DI. Clinical management of endometriosis. Obstet Gynecol;118:691-705.

20. Hudelist G, English J, Thomas AE, Tinelli A, Singer $\mathrm{CF}$, Keckstein J. Diagnostic accuracy of transvaginal ultrasound for non-invasive diagnosis of bowel endometriosis: systematic review and meta-analysis. Ultrasound Obstet Gynecol 2011;37:257-63. 